\title{
Stereocilin gene variants associated with episodic vertigo: expansion of the DFNB16 phenotype
}

\author{
Carina Frykholm ${ }^{1} \cdot$ Joakim Klar $\mathbb{B}^{1,2} \cdot$ Tatjana Tomanovic $^{3} \cdot$ Adam Ameur $^{2} \cdot$ Niklas Dahl $^{1,2}$
}

Received: 14 March 2018 / Revised: 7 August 2018 / Accepted: 19 August 2018 / Published online: 24 September 2018

(c) European Society of Human Genetics 2018

\begin{abstract}
Vestibular disorders comprise a heterogeneous group of diseases with transient or permanent loss of vestibular function. Vestibulopathy is in most cases associated with migraine, Ménière disease, hereditary ataxias, or sensorineural hearing loss. We identified two brothers and their first cousin affected by hearing loss and episodic vertigo. The brothers were homozygous STRC nonsense variant [c.4027 C > T, p. (Q1343*)], whereas their first cousin was compound heterozygous for the STRC nonsense variant and a $97 \mathrm{~kb}$ deletion spanning the entire STRC gene. Clinical investigations confirmed pathological vestibular responses in addition to a characteristic DFNB16 hearing loss. The STRC gene encodes Stereocilin in the cochlea and in the vestibular organ where it ensheathes the kinocilium of the otolithic membranes. Stereocilin is associated with the gel overlaying the vestibular kinocilia, suggesting a role for the protein in sensing balance and spatial orientation. Our findings support such a function for Stereocilin in the vestibular organ and expand the phenotype associated with DFNB16.
\end{abstract}

\section{Introduction}

The vestibular organ comprises the semicircular canals to sense rotation, and the otolithic organs, the utricle and the saccule, required to sense linear acceleration. Vestibular disorders are usually episodic and may involve one or several symptoms such as vertigo, motion sickness, dizziness, nystagmus, blurred vision, and unsteadiness $[1,2]$. Vestibulopathy is, in most cases, part of cochlear-

These authors contributed equally: Carina Frykholm and Joakim Klar.

Electronic supplementary material The online version of this article (https://doi.org/10.1038/s41431-018-0256-6) contains supplementary material, which is available to authorized users.

$\triangle$ Niklas Dahl

niklas.dahl@igp.uu.se

1 Department of Immunology, Genetics and Pathology, Uppsala University and Uppsala University Hospital, Uppsala, Sweden

2 Department of Immunology, Genetics and Pathology, Science for Life Laboratory, Biomedical Centre, Uppsala University, Uppsala, Sweden

3 Department of Hearing and Balance Disorders, Karolinska Hospital, Solna, Sweden vestibular disorders with hearing loss, tinnitus, and ataxia [3-5].

Variants in STRC cause DFNB16B (MIM\# 60372) representing at least $10 \%$ of cases with autosomal recessive, non-syndromic sensorineural hearing loss (SNHL) [6-8]. Deletions spanning the $S T R C$ gene has an estimated carrier frequency of $1.6 \%$ in a European population [9]. In mice, stereocilin is expressed in the kinocilia of the vestibular saccule and utricle, and to a lower extent in the ampullas of the semicircular canals in addition to the expression in the cochlea $[10,11]$. However, the clinical significance of Stereocilin in the vestibular organ has remained elusive $[6,8,11]$.

\section{Methods}

\section{Subjects}

We identified a non-consanguineous family of SwedishNorwegian origin segregating two brothers (III:2 and III:4) and their first cousin (III:6) with nonprogressive and moderate SNHL (Fig. 1a, b). The affected members had a history of episodic vertigo from age 2 years (III:2), 5 years (III:4), and 11 years (III:6), respectively. 


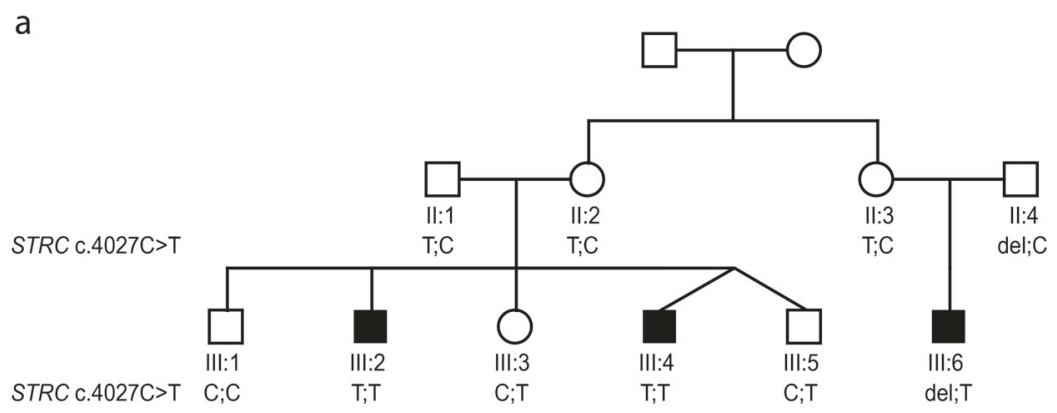

C

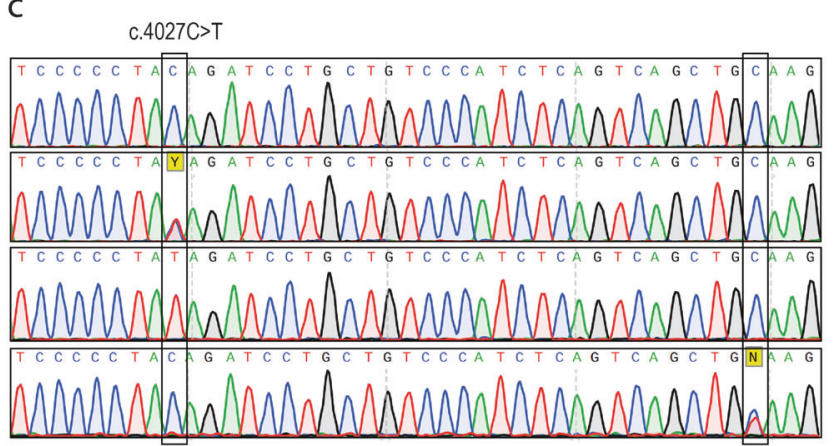

Fig. 1 Pedigree of the family segregating DFNB16 and episodic vertigo. a Pedigree indicating three members affected by moderate sensorineural hearing loss and episodic vertigo. Segregation of the STRC variants is shown below each symbol. b Bilateral tone audiograms from the three affected individuals homozygous, or compound heterozygous, for the STRC variants. c Chromatograms showing part of $S T R C$ exon 20 using STRC specific primers from a non-carrier (top row), a heterozygous individual (second row), and an individual

\section{Vestibular tests}

Vestibular function was assessed using bithermal caloric test, vestibular head impulse (vHIT), videonystagmogram, subjective visual horizontal (SVH) test, cervical (c) and ocular (o) vestibular-evoked myogenic potential (VEMP) according to standardized methods [12].

\section{Exome sequencing and SNP array analysis}

Exome sequencing was performed on DNA from two affected individuals (III:2 and III:4) on the Ion Proton System (Life Technologies, Carlsbad, CA, USA; Supplementary Information). CytoScan high-density array (Thermo Fisher Scientific, Waltham, MA) analysis was performed on DNA from III:6 and his unaffected parents using computer software Chromosome Analysis Suite version 3.3 (Thermo Fisher Scientific). The DNA variants identified in this study are accessible in the dbSNP: https://www.ncbi.nlm.nih.gov/SNP/ and the ClinVar database: https://www.ncbi.nlm.nih.gov/ clinvar/. b

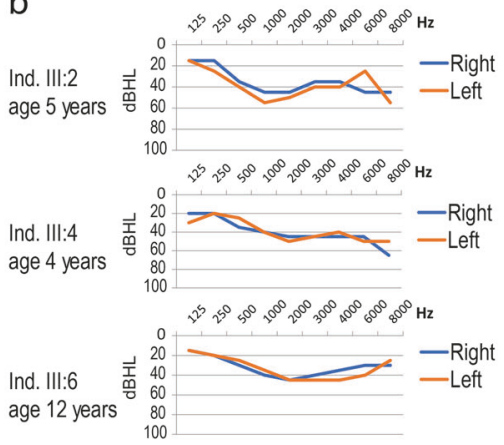

d

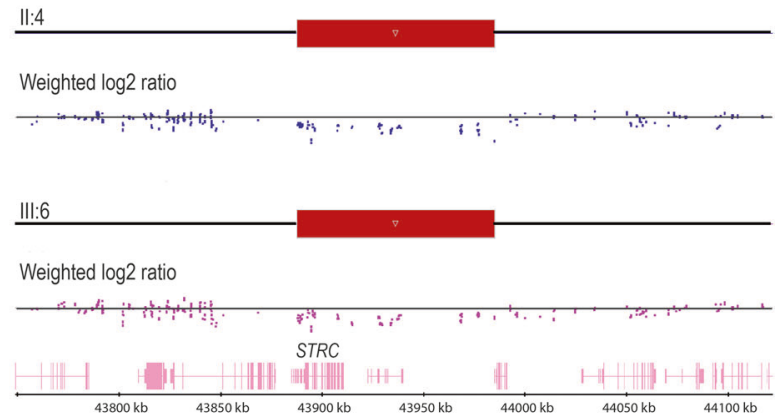

homozygous for the c. $4027 \mathrm{C}>\mathrm{T}$ nonsense variant (third row), respectively. The bottom chromatogram is from a non-carrier using primers that anneal to both STRC and $p S T R C$. The position with a sequence divergence between STRC and pSTRC (c.3931-81) is boxed. d Cytoscan HD Array analysis showing the heterozygous $97 \mathrm{~kb}$ deletion (red box) spanning STRC in ind. III:6 and his unaffected father (II:4). del: $97 \mathrm{~kb}$ deletion spanning the entire $S T R C$ gene

\section{Results and discussion}

Individual III:2 had an onset of episodic vertigo at 2 years of age characterized by imbalance, blurred vision, vomiting, and nausea. The frequency of episodes was one to four annually and became less frequent from 15 years of age. Individual III:4 had episodes of positional vertigo associated with vomiting since the age of 5 years. With age, the episodes became less intense. Individual III:6 had an onset of episodes of vertigo associated with nausea, vomiting, and blurred vision at 11 years of age. These episodes occurred frequently the first year but became rare from age 12 years. In all three affected individuals, the episodes lasted for one to several days and were triggered by food or sleep deprivation. No other neurological symptoms or cognitive abnormalities were identified.

The two brothers underwent a detailed vestibular examination at age 8 years and 16 years (III:2), and at age 12 years (III:4), respectively (Table 1, Supplementary Table 1). In III:2, bilateral bithermal caloric stimulation revealed a left-sided weakness with a ratio of 0.25 at 8 years of age. This was normalized at age 16 years. However, III: 2 had a 
Table 1 Vestibular test results in two individuals with DFNB16, who are homozygous for the $S T R C$ variant c.4027C $>$ T

\begin{tabular}{|c|c|c|c|c|c|c|c|c|c|c|}
\hline \multirow[t]{2}{*}{ Subject } & \multicolumn{10}{|c|}{ Test (normal ranges) } \\
\hline & vHIT $(0.8-1)$ & & $\begin{array}{l}\text { Caloric } \\
\text { ratio } \\
(0-0.2)\end{array}$ & $\begin{array}{l}\text { SVH upright } \\
(-2.5 M>+2.5)\end{array}$ & $\begin{array}{l}\text { SVH tilt } \\
(>-3)\end{array}$ & & $\begin{array}{l}\text { o-VEMP } \\
\text { (Amplitude diff. 2.5) }\end{array}$ & & $\begin{array}{l}\text { o-VEMP (Amplitude diff. 2.5)c- } \\
\text { VEMP (Amplitude diff. } 2.5 \text { ) }\end{array}$ & \\
\hline III:2 & R: 0.94 & L: 0.95 & $0.25^{\mathrm{a}}$ & -0.38 & $\mathrm{R}: 2.5$ & $\mathrm{~L}: 16^{\mathrm{b}}$ & $\mathrm{R}: 3$ & L: 0 & $\mathrm{R}: 1.1$ & L: 0.9 \\
\hline III:4 & $\mathrm{R}: 0.87$ & L: 0.85 & 0.02 & 0.15 & $\mathrm{R}:-8^{\mathrm{c}}$ & $\mathrm{L}:-16^{\mathrm{c}}$ & $\mathrm{R}: 2.4$ & L: 1.4 & R: 0.7 & L: $0.2^{\mathrm{d}}$ \\
\hline
\end{tabular}

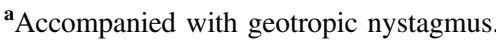

${ }^{\mathbf{b}}$ Right vs. left difference indicates left-sided utricle asymmetry for tilt perception.

${ }^{\mathbf{c}}$ Pathological values for tilt perception.

d c-VEMP with acoustic stimulation revealed significant amplitude difference.

$c$-VEMP cervical vestibular-evoked myogenic potential, $L$ left, $o$-VEMP ocular vestibular-evoked myogenic potential, $R$ right, $S V H$ subjective visual horizontal test, $v H I T$ vestibular head impulse

persistent positional left-sided and slow geotropic nystagmus at both occasions suggesting a primary labyrinthine asymmetry and not an otolith detachment causing benign and paroxysmal positional vertigo. In supine position and after headshake, nystagmus was directed to the left. cVEMP revealed similar amplitudes on both sides after correction, whereas o-VEMP amplitudes with acoustic stimulation showed a side difference $[13,14]$. The vHIT test and SVH in upright position were within normal range ($2.5\langle M\rangle+2.5)$. However, when SVH was measured at tilted body positions, the mean value $(K)$ on the right side was 2.5 and on the left side 16 ( $>-3$ is normal), indicating a left-sided utricle asymmetry for tilt perception [15]. Furthermore, the left vertical vestibulo-ocular reflex was weak, consistent with an otolithic disorder. Caloric stimulations on III:4 revealed a normal ratio (0.02). The o-VEMP amplitudes were symmetrical, suggesting normal function of the superior vestibular nerve or utricule. However, c-VEMP with acoustic stimulation showed amplitude difference, indicating a weakness of the saccule or inferior nerve. The vHIT showed a normal bilateral gain. An SVH in upright position were within the normal range $(-2.5<M\rangle+2.5)$, whereas investigation of tilt perceptions revealed a pathological mean value $(K)$ on both sides (right: -8 ; left: -16 ). The left vertical vestibulo-ocular reflex was weak. In summary, the results from vestibular tests indicated a left-sided utricular weakness in III:2, whereas III:4 had a bilateral and predominantly left-sided dysfunction of the vestibular organ involving both the saccular and utricular parts. III:6 was reluctant to vestibular testing but his detailed description of episodes, confirmed by his parents, was consistent with the vestibular symptoms in III:2 and III:4.

Whole exome sequencing was performed on DNA from two affected individuals (III:2 and III:4). The analysis identified two rare homozygous variants (1\% cutoff) shared by the two brothers: A nonsense STRC variant (NM_153700.2; rs144948296, ClinVar accession RCV000516500.1, c.4027
$\left.\mathrm{C}>\mathrm{T}, \quad \mathrm{p} .\left(\mathrm{Q} 1343^{*}\right)\right)$ and a missense $P C N T$ variant (NM_006031.5; rs35044802, c.3580 G > A, p.(A1194T)). A non-processed pseudogene ( $p$ STRC) with a $99.6 \%$ coding homology to the structural STRC gene complicates the identification of variants. A natural sequence divergence between STRC and $p S T R C$ [NG_011636.1(NM_153700.2): c.3931-81 T >C, rs2467434] was used to design STRC specific primers (Fig. 1a, c; Supplementary information). Analysis of the first cousin (III:6) revealed compound heterozygosity for the STRC variant c. $4027 \mathrm{C}>\mathrm{T}$ inherited from the mother and a deletion of approximately $97 \mathrm{~kb}$ [ClinVar submission ID SUB4337996, chr15:g.(438868571_ 43888004)_(43984930_43992627)del(hg19] spanning the STRC, CKMTIB and CATSPER 2 genes, inherited from the father (Fig. 1d). The PCNT variant is present in dbSNP (rs35044802) and the Genome Aggregation Database (gno$\mathrm{mAD}$ ) [16]. The variant is predicted to be benign (PolyPhen2 score 0.016 benign; PROVEAN score -0.02 neutral; Sift 0.718 tolerated) and was not further considered. The STRC variant $c .4027 \mathrm{C}>\mathrm{T}$ was predicted to be damaging by Mutation Taster and has previously been identified in a heterozygous state in combination with a STRC deletion on the second allele in a DFNB16 patient without reported vestibular symptoms [17].

Balance problems, but not vestibulopathy, have been observed in a few reported DFNB16 families $[18,19]$. In these studies, vestibular function was either not mentioned or investigated. It cannot be excluded that infrequent and/or mild vestibular symptoms have remained undiagnosed. The sense of balance depends on information from vestibular organs, proprioceptive sensors, and visual impression to the cerebellum. In unilateral canal paresis (lateral vestibular semicircular canal), detected as an asymmetry in the caloric response, a central compensation will occur [20]. With time, the vestibular symptoms fade away and symmetry in caloric response is restored (Supplementary Table 2, II:2). The time span for central compensation in vestibular dysfunction is 
shorter in children than in adults and probably holds for other semicircular canals and the otolith organs. In mice vestibular organ, Stereocilin is predominantly expressed in the saccule and utricle. A similar expression pattern in humans may explain the effect on the otolith organs in our patients. Both siblings are right-handed consistent with central projections on the right vestibular organ. The recorded aberrations on the left side may be explained by a less effective left-sided central compensation [20]. Balance or behavior abnormalities have not been reported in $\mathrm{Strc}^{-/-}$ mice. However, vestibular testing in the mouse model has not been performed and a mild, transient, or compensated vestibulopathy cannot be excluded. Furthermore, phenotypes in mice may be strain-specific and with compensatory mechanisms that are not present in humans. In humans, symptoms from vestibular dysfunction are heterogeneous including vertigo, dizziness, visual and balance symptoms, problems of emotion, memory, and self-perception. This may be difficult to describe, especially for children. In addition, strong compensatory mechanisms for vestibular deficiency in humans are well known [20]. Consequently, the episodic phenotype identified in our patients may have been overlooked in other DFNB16 patients. More extensive analysis of the Strc-null mouse and DFNB16 patients are now required to understand the role for Stereocilin in vestibular function.

In conclusion, we diagnosed episodic vertigo segregating DFNB16 and STRC variants affecting protein function in two brothers and their first cousin. Clinical investigations confirmed vestibular dysfunctions primarily from the saccula and utricule but less prominent in the semicircular canals, consistent with the distribution of Stereocilin in the vestibular organ [10]. Stereocilin is expressed in kinocilia of the vestibular hair cells and at the tips of individual hair cells, suggesting a function for the protein in the coupling of vestibular kinocilia to the otoconial membrane, required to sense both orientation and motion. Our report thus expands the DFNB16 phenotype and provides an additional mechanism for episodic vertigo.

\section{Ethical approval}

This study was approved by the regional ethical committee of Uppsala, Sweden (Dnr 2004:M-270).

Acknowledgements We thank the patients and their family for cooperation. This work was supported by grants from the Swedish Research Council (2015-02424 to ND), Hjärnfonden, and Science for Life Laboratory, Sweden.

\section{Compliance with ethical standards}

Conflict of interest The authors declare that they have no conflict of interest.

\section{References}

1. Bisdorff A, Bosser G, Gueguen R, Perrin P. The epidemiology of vertigo, dizziness, and unsteadiness and its links to co-morbidities. Front Neurol. 2013;4:29.

2. Brantberg K. Familial early-onset progressive vestibulopathy without hearing impairment. Acta Otolaryngol. 2003;123:713-7.

3. Eppsteiner RW, Smith RJ. Genetic disorders of the vestibular system. Curr Opin Otolaryngol Head Neck Surg. 2011;19:397402.

4. Requena T, Espinosa-Sanchez JM, Lopez-Escamez JA. Genetics of dizziness: cerebellar and vestibular disorders. Curr Opin Neurol. 2014;27:98-104.

5. Frejo L, Giegling I, Teggi R, Lopez-Escamez JA, Rujescu D. Genetics of vestibular disorders: pathophysiological insights. J Neurol. 2016;263(Suppl 1):S45-53.

6. Francey LJ, Conlin LK, Kadesch HE, et al. Genome-wide SNP genotyping identifies the Stereocilin (STRC) gene as a major contributor to pediatric bilateral sensorineural hearing impairment. Am J Med Genet A. 2012;158a:298-308.

7. Sloan-Heggen CM, Bierer AO, Shearer AE, et al. Comprehensive genetic testing in the clinical evaluation of 1119 patients with hearing loss. Hum Genet. 2016;135:441-50.

8. Vona B, Hofrichter MA, Neuner C, et al. DFNB16 is a frequent cause of congenital hearing impairment: implementation of STRC mutation analysis in routine diagnostics. Clin Genet. 2015;87: $49-55$.

9. Knijnenburg J, Oberstein SA, Frei K, et al. A homozygous deletion of a normal variation locus in a patient with hearing loss from non-consanguineous parents. J Med Genet. 2009;46:412-7.

10. Verpy E, Leibovici M, Michalski N, et al. Stereocilin connects outer hair cell stereocilia to one another and to the tectorial membrane. J Comp Neurol. 2011;519:194-210.

11. Verpy E, Masmoudi S, Zwaenepoel I, et al. Mutations in a new gene encoding a protein of the hair bundle cause non-syndromic deafness at the DFNB16 locus. Nat Genet. 2001;29:345-9.

12. Tribukait A, Brantberg K, Bergenius J. Function of semicircular canals, utricles and saccules in deaf children. Acta Otolaryngol. 2004;124:41-48.

13. Brantberg K, Bergenius J, Tribukait A. Vestibular-evoked myogenic potentials in patients with dehiscence of the superior semicircular canal. Acta Otolaryngol. 1999;119:633-40.

14. Holmeslet B, Foss OA, Bugten V, Brantberg K. Ocular vestibularevoked myogenic potentials (oVEMPs) in response to boneconducted vertex vibration. Clin Neurophysiol. 2015;126:608-13.

15. Tribukait A. Subjective visual horizontal in the upright posture and asymmetry in roll-tilt perception: independent measures of vestibular function. J Vestib Res Equilib Orientat. 2006;16:35-43.

16. Lek M, Karczewski K, Minikel E, et al. Analysis of proteincoding genetic variation in 60,706 humans. 2016;536:285-91.

17. Mandelker D, Amr SS, Pugh T, et al. Comprehensive diagnostic testing for stereocilin: an approach for analyzing medically important genes with high homology. J Mol Diagn. 2014;16: 639-47.

18. Villamar M, del Castillo I, Valle N, Romero L, Moreno F. Deafness locus DFNB16 is located on chromosome 15q13-q21 within a 5-cM interval flanked by markers D15S994 and D15S132. Am J Hum Genet. 1999;64:1238-41.

19. Zhang Y, Malekpour M, Al-Madani N, et al. Sensorineural deafness and male infertility: a contiguous gene deletion syndrome. J Med Genet. 2007;44:233-40.

20. Zwergal A, Schlichiger J, Xiong G, et al. [(18)F]FDG uPET whole-brain imaging of central vestibular compensation: a model of deafferentation-indiced brain plasticity. Brain Struct Funct. 2016;221:159-70. 